\title{
Lagern Sie richtig?
}

Steinschnittlagerung Im letzten Teil unserer Serie zeigen wir Ihnen die Steinschnittlagerung. Der Begriff ist historisch bedingt und stammt aus der Urologie, da in dieser Patientenlagerung Blasensteine entfernt wurden ${ }^{1}$ und werden. Auch dieses Mal interessiert uns wieder: Wie führen Sie die Steinschnittlagerung in Ihrem OP durch? Senden Sie uns Ihre Tipps und Erfahrungen per E-Mail anImOP@thieme.de.

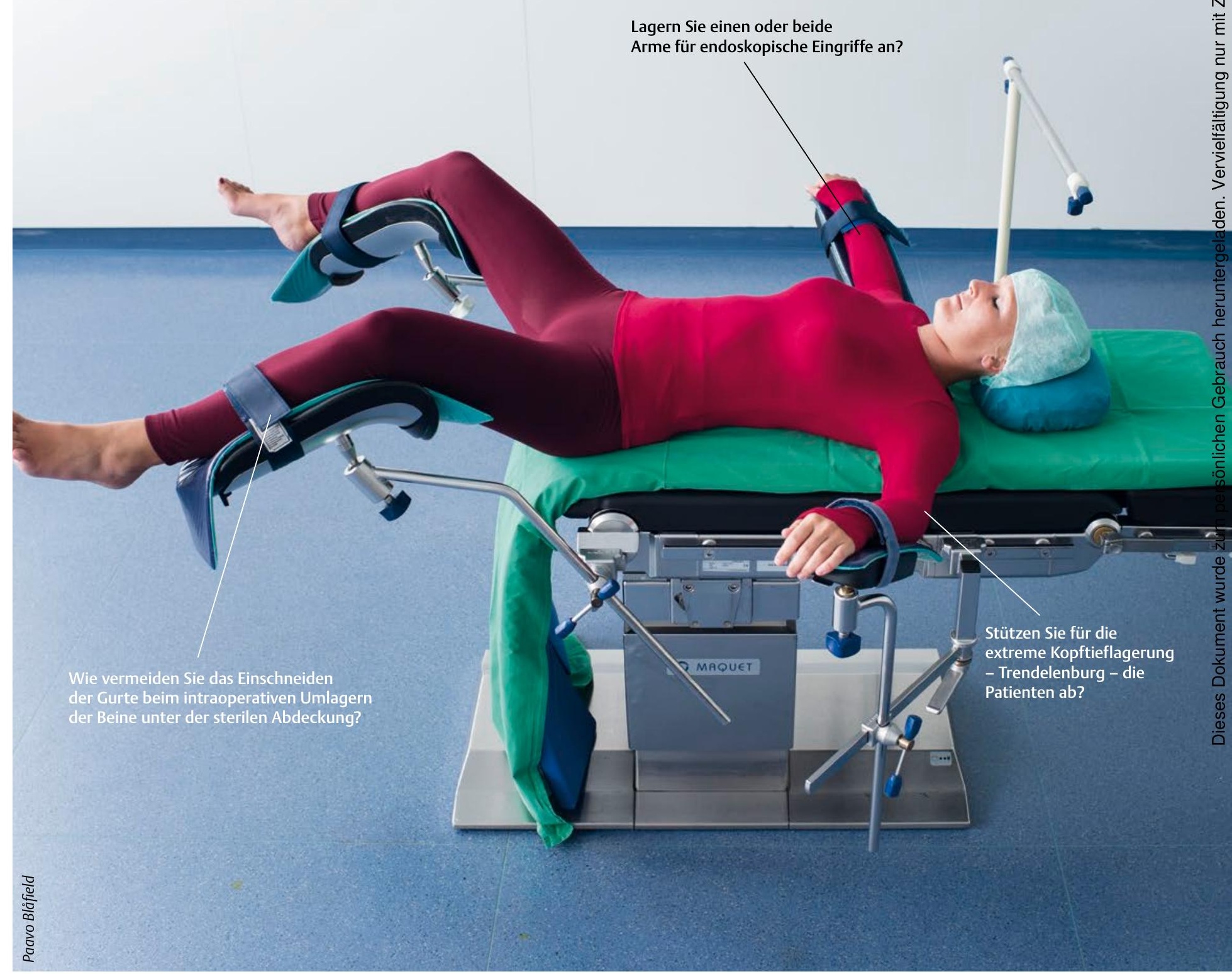

An den mit Pfeilen markierten Stellen besteht Diskussionsbedarf. Deshalb würden wir gern von Ihnen wissen, wie Sie die Steinschnittlagerung an Ihrer Klinik durchführen? 
- Karl-Heinz Wagenhäuser-

Patienten, die in der sogenannten Steinschnittlage (SSL) gelagert werden, kommen aus den Fachbereichen Gynäkologie, Urologie, Proktologie oder auch Viszeralchirurgie.

Der Patient liegt hierbei auf dem Rücken, die Beine sind um zirka 90 Grad in den Hüften gebeugt, die Knie abgewinkelt, sodass die Unterschenkel oft in den sogenannten „Göbelbeinstützen“ leicht abgespreizt gelagert werden.

Diese Göbelbeinstützen werden am OP-Tisch mithilfe von Kloben angebracht, die entweder per Hand oder über den OPTisch selbst verstellbar sind.

Bei dem Hochlagern der Beine kann es zu Läsionen des Nervus femoralis oder Nervus peronaeus sowie zu einem Kompartmentsyndrom des Unterschenkels kommen. Risikofaktoren sind außerdem lange Lagerungszeiten und starke Beugung und Rotation im Hüftgelenk.

Die Lagerung muss im Wachzustand vorgenommen werden, wenn Kontrakturen oder Hüftgelenkserkrankungen vorliegen.

Die ursprüngliche Steinschnittlage wird für eine offene oder endoskopische OP angepasst, die Beine sind dann flacher (nicht in 90 Grad) gelagert. Dieses Absenken der Beine sollte nicht ausschließlich über die „Leg-Down“-Taste vorgenommen werden, sondern per Hand über die verstellbaren Kloben. Die vorgegebene Form der Göbelbeinstütze kann dann besser anmodelliert und Lagerungsschäden können von vornherein minimiert werden.

Ist eine Lagerungsänderung während der OP geplant, sollte eine Probefahrt zum Verändern der Lagerung vor dem sterilen Abdecken durchgeführt werden, da so die Sichtkontrolle durchgeführt werden kann. Dabei sollte darauf geachtet werden, ob der Sicherungsgurt drückt oder sich die Stützen in die Kniekehlen schieben und die oben beschriebenen Lagerungsschäden verursachen.

Wenn bei endoskopischen Eingriffen eine extreme Kopftieflagerung - Trendelenburg Lagerung - vorgenommen wird, sollte das „vom Tisch Rutschen des Patienten“ durch geeignete Maßnahmen, wie gepolsterte Schulterstützen oder eine Teilvakuummatratze mit anmodellierter Schulterpartie, verhindert werden.
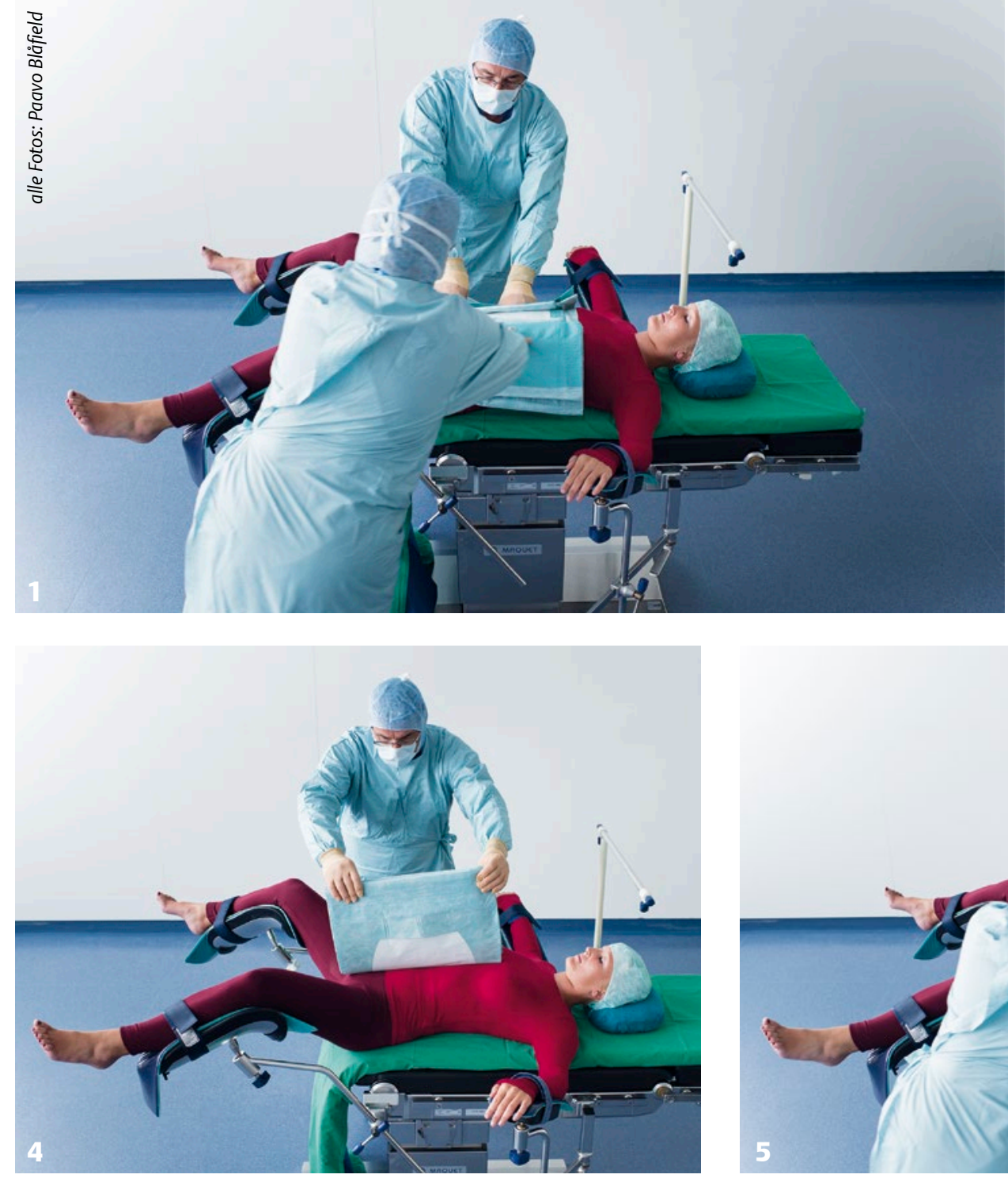

5
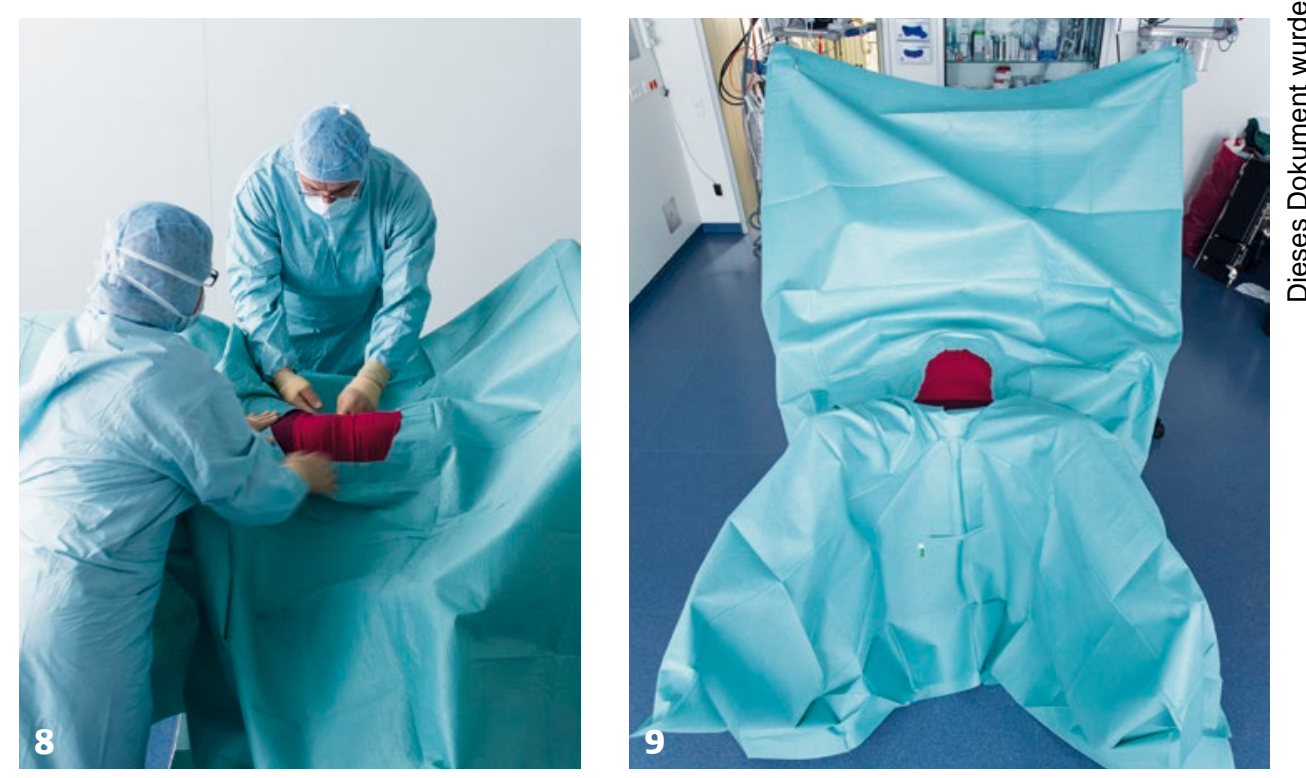
